\title{
The Hodge Numbers of the Moduli Spaces of Vector Bundles over a Riemann Surface
}

\author{
Richard Earl and Frances Kirwan
}

October 16, 2018

\section{Introduction}

Let $\mathcal{M}(n, d)$ denote the moduli space of stable holomorphic vector bundles of coprime rank $n$ and degree $d$ over a fixed Riemann surface $\Sigma$ of genus $g \geq 2$. Let $\Lambda$ be a fixed line bundle over $\Sigma$ of degree $d$ and let $\mathcal{M}_{\Lambda}(n, d) \subset \mathcal{M}(n, d)$ denote the space consisting of those bundles with determinant $\Lambda$. The spaces $\mathcal{M}(n, d)$ and $\mathcal{M}_{\Lambda}(n, d)$ are nonsingular complex projective varieties whose geometry has been much studied. In particular Harder, Narasimhan, Desale and Ramanan first described in 1975 an inductive method to determine the Betti numbers of $\mathcal{M}(n, d)$ using number theoretic methods and the Weil conjectures [12, 1]. Subsequently in 1982 Atiyah and Bott [1] obtained the same inductive method using gauge theory.

In this note we give a similar inductive method for determining the Hodge-Poincaré polynomials of $\mathcal{M}(n, d)$ and $\mathcal{M}_{\Lambda}(n, d)$, that is

$$
H P(\mathcal{M}(n, d))(x, y)=\sum_{p \geq 0} \sum_{q \geq 0} h^{p, q}(\mathcal{M}(n, d)) x^{p} y^{q}
$$

and

$$
H P\left(\mathcal{M}_{\Lambda}(n, d)\right)(x, y)=\sum_{p \geq 0} \sum_{q \geq 0} h^{p, q}\left(\mathcal{M}_{\Lambda}(n, d)\right) x^{p} y^{q}
$$

where $h^{p, q}$ denote the Hodge numbers (see Theorem 1 and Lemma 3 below). The $\chi(t)$ characteristic of $\mathcal{M}_{\Lambda}(n, d)$, that is $\chi(t)=H P\left(\mathcal{M}_{\Lambda}(n, d)\right)(t,-1)$, has an especially simple form (see Corollary 6), while the $\chi(t)$-characteristic of $\mathcal{M}(n, d)$ is identically zero.

In [1] Atiyah and Bott identify $\mathcal{M}(n, d)$ with the quotient of $\mathcal{C}^{s}$, the infinite dimensional space of stable holomorphic structures on a fixed $C^{\infty}$ complex bundle $\mathcal{E}$ of rank $n$ and degree $d$ over $\Sigma$, by $\mathcal{G}_{c}$, the infinite dimensional group of smooth complex automorphisms. They introduce a stratification for $\mathcal{C}$, the infinite dimensional affine space of all holomorphic structures on $\mathcal{E}$, which is equivariantly perfect with respect to the action of $\mathcal{G}_{c}$ (or equivalently the gauge group $\mathcal{G}$ ) and which has $\mathcal{C}^{s}$ as an open stratum. The resulting Morse equalities give the equivariant cohomology of the stable stratum and thus the cohomology of $\mathcal{M}(n, d)$, in terms of the classifying space of $\mathcal{G}$ and the equivariant cohomology of the unstable strata, which can be calculated inductively.

In [14 the methods of Atiyah and Bott are adapted to finite dimensional quotients in the sense of Mumford's geometric invariant theory (GIT) [17, 19], considering the linear 
action of a complex reductive group on a nonsingular complex projective variety where every semistable point is stable. This method generalises to give an inductive approach determining the Hodge numbers [14, §14].

To apply this to the case of $\mathcal{M}(n, d)$ we recall from Newstead [19] how this moduli space may be expressed as a finite dimensional quotient in the sense of GIT. It is shown in 15] that the resulting finite dimensional stratification coming from [14] corresponds naturally to the stratification of Atiyah and Bott outside a subset whose codimension tends to infinity with $d$, and the equivariant cohomology of the corresponding strata agrees up to a degree tending to infinity with $d$. Since $\mathcal{M}(n, d)$ depends on $d$ only through its remainder modulo $n$, we may then refine Atiyah and Bott's inductive formulas for the Betti numbers to give the Hodge numbers as well.

In the case $n=2$ our formula for the Hodge numbers of $\mathcal{M}_{\Lambda}(n, d)$ was recently proved by del Baño Rollin [3, §3] and had been independently discovered by Newstead [21]. The formula for the $\chi(t)$-characteristic of $\mathcal{M}_{\Lambda}(2,1)$ was first proved by Narasimhan and Ramanan [18] in 1975.

The layout of this paper is as follows. In Section 1 we review the arguments used in [1] and [15 to obtain inductive formulas for the Betti numbers of $\mathcal{M}_{\Lambda}(n, d)$. In Section 2 we adapt those arguments to Hodge numbers and prove our main result Theorem 11. In Section 3 we explicitly calculate $\operatorname{HP}\left(\mathcal{M}_{\Lambda}(n, d)\right)(x, y)$ for $n=2,3$. In Section 4 we discuss the $\chi(t)$-characterstic and explain how $\chi(t)$ contains information about intersection pairings involving certain generators of $H^{*}\left(\mathcal{M}_{\Lambda}(n, d)\right)$.

\section{The Two Approaches}

Let $\mathcal{E}$ be a fixed $C^{\infty}$ complex bundle of rank $n$ and degree $d$ over $\Sigma$ and let $\mathcal{C}$ denote the infinite dimensional affinespace of holomorphic structures on $\mathcal{E}$. For each holomorphic bundle $E \in \mathcal{C}$ there is a strictly ascending canonicalfiltration [12, p.221]

$$
0=E_{0} \subset E_{1} \subset \cdots \subset E_{P-1} \subset E_{P}=E
$$

such that the quotients $Q_{j}=E_{j} / E_{j-1}$ are semistable and such that

$$
\frac{\operatorname{deg}\left(Q_{j}\right)}{\operatorname{rk}\left(Q_{j}\right)}=\mu\left(Q_{j}\right)>\mu\left(Q_{j+1}\right)=\frac{\operatorname{deg}\left(Q_{j+1}\right)}{\operatorname{rk}\left(Q_{j+1}\right)} \text {. }
$$

We then say that $E$ has type

$$
\mu=\left(\mu\left(Q_{1}\right), \ldots, \mu\left(Q_{P}\right)\right) \in \mathbf{Q}^{n}
$$

where $\mu\left(Q_{j}\right)$ appears $\operatorname{rk}\left(Q_{j}\right)$ times. Atiyah and Bott defined a stratification $\left\{\mathcal{C}_{\mu}: \mu \in \mathcal{M}\right\}$ by setting $\mathcal{C}_{\mu} \subset \mathcal{C}$ to be the set of all holomorphic bundles $E$ over $\Sigma$ of type $\mu$. The set $\mathcal{C}^{s s}$ of semistable bundles is precisely the stratum of type

$$
\mu_{0}=\left(\frac{d}{n}, \cdots, \frac{d}{n}\right) .
$$

This stratification $\left\{\mathcal{C}_{\mu}: \mu \in \mathcal{M}\right\}$ is equivariantly perfect with respect to the action of the gauge group $\mathcal{G}$. Thus if we let

$$
P_{G}(X, t)=\sum_{j \geq 0} t^{j} \operatorname{dim}_{\mathbf{Q}} H_{G}^{j}(X, \mathbf{Q})
$$


denote the equivariant Poincaré polynomial of a space $X$ acted on by a group $G$, then we have equivariant Morse equalities

$$
P_{\mathcal{G}}(\mathcal{C})(t)=P_{\mathcal{G}}\left(\mathcal{C}^{s s}\right)(t)+\sum_{\mu \neq \mu_{0}} t^{2 d_{\mu}} P_{\mathcal{G}}\left(\mathcal{C}_{\mu}\right)(t)
$$

where $d_{\mu}$ is the complex codimension of $\mathcal{C}_{\mu}$ in $\mathcal{C}$ which is given by the formula [1, 7.16]

$$
d_{\mu}=\sum_{1 \leq j<i \leq P} n_{i} d_{j}-n_{j} d_{i}+n_{i} n_{j}(g-1) .
$$

The $\mathcal{G}$-equivariant cohomology of the strata may be determined inductively via the isomorphisms [1], 7.12]

$$
H_{\mathcal{G}}^{*}\left(\mathcal{C}_{\mu}\right) \cong \bigotimes_{1 \leq j \leq P} H_{\mathcal{G}\left(n_{j}, d_{j}\right)}^{*}\left(\mathcal{C}\left(n_{j}, d_{j}\right)^{s s}\right)
$$

The $\mathcal{G}$-equivariant Poincaré polynomial of the affine space $\mathcal{C}$ is given by [1], Thm. 2.15]

$$
P_{\mathcal{G}}(\mathcal{C})(t)=\frac{\prod_{l=1}^{n}\left(1+t^{2 l-1}\right)^{2 g}}{\left(1-t^{2 n}\right) \prod_{l=1}^{n-1}\left(1-t^{2 l}\right)^{2}}
$$

Using this Atiyah and Bott obtain an inductive formula

$$
P_{\mathcal{G}}\left(\mathcal{C}^{s s}\right)(t)=P_{\mathcal{G}}(\mathcal{C})(t)-\sum_{\mu \neq \mu_{0}} t^{2 d_{\mu}} P_{\mathcal{G}\left(n_{j}, d_{j}\right)}\left(\mathcal{C}\left(n_{j}, d_{j}\right)^{s s}\right)(t)
$$

(where the sum is over all unstable types $\mu=\left(d_{1} / n_{1}, \ldots, d_{P} / n_{P}\right)$ ) for the $\mathcal{G}$-equivariant Betti numbers of $\mathcal{C}^{s s}$. They show that when $n$ and $d$ are coprime the quotient $\overline{\mathcal{G}}=\mathcal{G} / S^{1}$ of $\mathcal{G}$ by its central subgroup, consisting of multiplication by scalars in $S^{1}$, acts freely on $\mathcal{C}^{s s}$ and that [1], 9.3]

$$
P(\mathcal{M}(n, d))(t)=P_{\overline{\mathcal{G}}}\left(\mathcal{C}^{s s}\right)(t)=\left(1-t^{2}\right) P_{\mathcal{G}}\left(\mathcal{C}^{s s}\right)(t) .
$$

Finally they show that [1, Prop. 9.7]

$$
P(\mathcal{M}(n, d))(t)=(1+t)^{2 g} P\left(\mathcal{M}_{\Lambda}(n, d)\right)(t) .
$$

The GIT approach described in 19 involves the action of a projective general linear group on a variety $R$ which naturally parametrises holomorphic bundles over $\Sigma$ as follows. For any stable bundle $E$ of rank $n$ and degree $d$ over $\Sigma$ of slope $d / n>2 g-1$ we have from [19, 5.2] and the Riemann-Roch theorem that

$$
E \text { is generated by its sections }
$$

and that

$$
\operatorname{dim} H^{0}(\Sigma, E)=d+n(1-g) \quad \text { and } \quad H^{1}(\Sigma, E)=0 .
$$

Let

$$
p=d+n(1-g)
$$

and let $G(n, p)$ denote the Grassmannian of $n$-dimensional quotients of $\mathbf{C}^{p}$. There is then a natural one-to-one correspondence between holomorphic maps

$$
f: \Sigma \rightarrow G(n, p)
$$


and holomorphic quotients $E$ of $\Sigma \times \mathbf{C}^{p}$ of rank $n$, defined by setting the fibre $E_{x}$ to be $f(x)$; more precisely $E=f^{*} Q$ where $Q$ is the tautological bundle of rank $n$ over the quotient Grassmannian $G(n, p)$.

Let $\operatorname{Hol}_{d}(\Sigma, G(n, p))$ denote the space of holomorphic maps $f: \Sigma \rightarrow G(n, p)$ such that the induced bundle $E=f^{*} Q$ has degree $d$. We then define $R \subset \operatorname{Hol}_{d}(\Sigma, G(n, p))$ to consist of those holomorphic maps such that $E=f^{*} Q$ satisfies $H^{1}(\Sigma, E)=0$ and the map on sections

$$
\mathbf{C}^{p} \rightarrow H^{0}(\Sigma, E)
$$

is surjective. $\mathrm{R}$ is then a nonsingular quasi-projective variety [15, p.265].

We now recall some properties of the action of $P G L(p)$ on Rand define a linearisation for this action. Assume that $d / n>2 g-1$. Then there is a quotient $\tilde{E}$ of the rank $p$ trivial complex bundle over $R \times \Sigma$ such that

(a) $\tilde{E}$ has the local universal property for families of bundles over $\Sigma$ of rank $n$ and degree $d$ which satisfy (5) and (6),

(b) for every $f \in R$ the restriction

$$
E^{f}=\left.\tilde{E}\right|_{\{f\} \times \Sigma}=f^{*} Q
$$

is the induced quotient of $\Sigma \times \mathbf{C}^{p}$,

(c) for $f, g \in R$ we have $E^{f} \cong E^{g}$ if and only if $f$ and $g$ lie in the same orbit of the action of $P G L(p)$ on $R$.

Now by [19, pp. 141-3] if $N$ is suitably large then $R$ can be embedded as a quasiprojective subvariety of $(G(n, p))^{N}$ by a map

$$
f \mapsto\left(f\left(x_{1}\right), \ldots, f\left(x_{N}\right)\right)
$$

where $x_{1}, \ldots, x_{N}$ are points of $\Sigma$. This provides the required linearisation for the action of $S L(p)$ on $R$. Moreover for sufficiently large $N$ and $d$ we have

(d) $f \in R^{s s}$ for this linearisation if and only if $E^{f}$ is semistable; further the closure of $R^{s s}$ in $(G(n, p))^{N}$ is in $R$.

When $n$ and $d$ are coprime integers, semistable bundles of rank $n$ and degree $d$ are stable and hence simple and thus $P G L(p)$ acts freely on $R^{s s}$. From GIT the quotient $R^{s s} / P G L(p)$ is a nonsingular complex projective variety which we have identified with $\mathcal{M}(n, d)$. Thus the Poincaré polynomial of $\mathcal{M}(n, d)$ is given by [15, 9.4]

$$
P_{P G L(p)}\left(R^{s s}\right)(t)=\left(1-t^{2}\right) P_{G L(p)}\left(R^{s s}\right)(t)
$$

It is proved in [15, Lemma 10.1] and [15, Cor. 7.4] that for any positive integer $k$ there is a natural isomorphism

$$
H_{G L(p)}^{j}(R) \cong H_{\mathcal{G}}^{j}(\mathcal{C})
$$

for $j \leq k$, provided that the degree $d$ is sufficiently large (depending on $k$ and $n$ ). We are always free to assume that the degree $d$ is arbitrarily large, because tensoring with a fixed line bundle over $\Sigma$ of degree $e$ induces an isomorphism

$$
\mathcal{M}(n, d) \rightarrow \mathcal{M}(n, d+n e) .
$$

Note that tensoring with such a line bundle takes semistable bundles to semistable bundles and takes bundles of type

$$
\mu=\left(d_{1} / n_{1}, \ldots, d_{P} / n_{P}\right)
$$


to bundles of type

$$
\mu+e=\left(d_{1} / n_{1}+e, \ldots, d_{P} / n_{P}+e\right) .
$$

Note also that by $(3)$ above, $P_{\mathcal{G}}(\mathcal{C})(t)$ is independent of $d$.

By [15, $\S 11]$ there is a stratification $\left\{S_{\beta}: \beta \in \mathcal{B}\right\}$ of $R$ which is the intersection with $R$ of the stratification of the product of Grassmannians $(G(n, p))^{N}$ described in [14, $\left.\S 16\right]$ and is closely related to the stratification $\left\{\mathcal{C}_{\mu}: \mu \in \mathcal{M}\right\}$ described above. In fact, to every possible type $\mu$ of a holomorphic bundle of rank $n$ and degree $d$ we can associate an index $\beta(\mu) \in \mathcal{B}[15,11.1]$. Moreover given any finite set $\mathcal{U}$ of types of bundles of rank $n$ and degree $d$, we can choose $N$ and $e$ sufficiently large that if $\mu \in \mathcal{U}+e$ then a point $f$ of $R$ lies in the stratum indexed by $\beta(\mu)$ if and only if the bundle $E^{f}$ is of type $\mu$ [15, Cor. 1.5]. We can also assume [15, Lemma 11.6], given any positive integer $k$ as above, that every stratum of $R$ not indexed by $\beta(\mu)$ for any $\mu \in \mathcal{U}+e$ has codimension greater than $k$ and that every stratum indexed by $\beta(\mu)$ for some $\mu \in \mathcal{U}+e$ has codimension the integer $d_{\mu}$ defined at (2) above, and equivariant Poincaré polynomial

$$
P_{G L(p)}\left(S_{\beta(\mu)}\right)(t)=\prod_{j=1}^{P} P_{G L\left(p_{j}\right)}\left(R\left(n_{j}, d_{j}\right)^{s s}\right)
$$

where $\mu=\left(d_{1} / n_{1}, \ldots, d_{P} / n_{P}\right)$ and $p_{j}=d_{j}+n_{j}(1-g)$ [15, Lemma 12.3]. Finally by [15, $§ 12]$ we can also assume that, outside a subset of codimension $k$, the stratification $\left\{S_{\beta}: \beta \in \mathcal{B}\right\}$ satisfies the conditions of [14, 9.5] to be equivariantly perfect. Putting all this together, we obtain

$$
P_{\mathcal{G}}(\mathcal{C})(t)=P_{G L(p)}\left(R^{s s}\right)(t)+\sum_{\mu \neq \mu_{0}} t^{2 d_{\mu}} \prod_{j=1}^{P} P_{G L\left(p_{j}\right)}\left(R\left(n_{j}, d_{j}\right)^{s s}\right)(t)+O\left(t^{k(n, d)}\right)
$$

where $k(n, d) \rightarrow \infty$ as $d \rightarrow \infty$ and $n$ remains fixed [15, §13]. This gives us an inductive formula which enables us to calculate the $G L(p)$-equivariant Betti numbers of $R^{s s}$, and thus the Betti numbers of $\mathcal{M}(n, d)$ when $n$ and $d$ are coprime, up to any arbitrarily large degree by using the isomorphism (9). It is, of course, equivalent to the inductive formula (4), but because its derivation involves stratifications of finite dimensional quasiprojective varieties instead of infinite dimensional spaces, we shall be able to apply [14, $\S 14]$ to generalise it to cover Hodge numbers as well as Betti numbers.

\section{Hodge Numbers}

In the last section we described inductive formulas from which the Betti numbers of the moduli spaces $\mathcal{M}(n, d)$ and $\mathcal{M}_{\Lambda}(n, d)$ can be calculated. We now want to show that these formulas can be refined to enable us to calculate the Hodge numbers of $\mathcal{M}(n, d)$ and $\mathcal{M}_{\Lambda}(n, d)$, as is done for GIT quotients of nonsingular projective varieties in [14, $\left.\S 14\right]$. As in [14, $\S 14]$ we have to make use of Deligne's extension of Hodge theory to complex quasi-projective varieties $Y$ which may be non-compact and singular [5, 6]. If $\mathrm{Y}$ is such a variety then its cohomology groups $H^{j}(Y ; \mathbf{C})$ have two canonical filtrations, the weight filtration and the Hodge filtration, giving a mixed Hodge structure on each $H^{j}(Y ; \mathbf{C})$. The Hodge numbers $h^{p, q}\left(H^{j}(Y)\right)$ of $H^{j}(Y)$ are then defined to be the dimensions of appropriate quotients associated to these filtrations [5, II 2.3.7] and satisfy

$$
\operatorname{dim} H^{j}(Y ; \mathbf{C})=\sum_{p, q} h^{p, q}\left(H^{j}(Y)\right) .
$$


Moreover $h^{p, q}\left(H^{j}(Y)\right)=0$ unless $p$ and $q$ lie between $\max \{0, j-m\}$ and $\min \{j, m\}$, where $m$ is the complex dimension of $Y$, and $p+q \leq j$ if $Y$ is projective whereas $p+q \geq j$ if $Y$ is nonsingular [5, III 8.2.9]. If $Y$ is both projective and nonsingular then the Hodge numbers $h^{p, q}\left(H^{j}(Y)\right)$ for $p+q=j$ are the classical Hodge numbers $h^{p, q}(Y)$. Let us define the Hodge-Poincaré polynomial of a quasi-projective variety $Y$ as

$$
H P(Y)(x, y, t)=\sum_{p, q, j} x^{p} y^{q} t^{j} h^{p, q}\left(H^{j}(Y)\right)
$$

Of course when $Y$ is projective and nonsingular we lose no information by setting $t=1$ and defining

$$
H P(Y)(x, y)=\sum_{p, q} x^{p} y^{q} h^{p, q}\left(H^{p+q}(Y)\right)
$$

we then have

$$
H P(Y)(x, y, t)=H P(Y)(x t, y t)
$$

We shall omit the variable $t$ when (11) is satisfied. We can also define equivariant Hodge numbers $h^{p, q}\left(H_{G}^{j}(Y)\right)$ when $Y$ is acted on algebraically by a complex reductive group $G$, by identifying $H_{G}^{*}(Y)$ with $H^{*}\left(Y \times_{G} E G\right)$ where $E G \rightarrow B G$ is a universal classifying bundle for $G$. Although $E G$ and $B G$ are not finite dimensional manifolds, there are natural Hodge structures on their cohomology (which in the case of $E G$ is of course trivial) (see [5, III $\S 9])$. We can regard $E G$ and $B G$ as increasing unions of finite dimensional varieties $(E G)_{m}$ and $(B G)_{m}$ for $m \geq 1$ such that $G$ acts freely on $(E G)_{m}$ with $(B G)_{m}=(E G)_{m} / G$ and the inclusions of $(E G)_{m}$ and $(B G)_{m}$ induce isomorphisms of cohomology in degrees less than $m$ which preserve the Hodge structure. For example

$$
B \mathbf{C}^{*}=\mathbf{C P}_{\infty}=\bigcup_{m \geq 1} \mathbf{C P}_{m}
$$

has Hodge-Poincaré polynomial

$$
H P\left(B \mathbf{C}^{*}\right)(x, y)=\sum_{j \geq 0} x^{j} y^{j}=\frac{1}{1-x y} .
$$

Similarly $Y \times_{G} E G$ is an increasing union of finite dimensional varieties whose mixed Hodge structures induce a natural mixed Hodge structure on the cohomology groups of $Y \times_{G} E G$. We want to generalise to Hodge numbers the arguments described in the last section to obtain an inductive formula giving the Betti numbers of $\mathcal{M}(n, d)$ using the identification of $\mathcal{M}(n, d)$ with the quotient $R^{s s} / G L(p)$. First recall that the equality (7) came from isomorphisms (cf. [15, $\S 9]$ and [11, $\S 9]$ )

$$
H_{P G L(p)}^{*}\left(R^{s s}\right) \cong H^{*}(\mathcal{M}(n, d))
$$

and

$$
H_{G L(p)}^{*}\left(R^{s s}\right) \cong H^{*}(\mathcal{M}(n, d)) \otimes H^{*}\left(B \mathbf{C}^{*}\right)
$$

The first of these isomorphisms comes from the fact that the central subgroup $\mathbf{C}^{*}$ of $G L(p)$ acts trivially on $R^{s s}$ and the induced action of the quotient $P G L(p)=G L(p) / \mathbf{C}^{*}$ is free. The natural map

$$
R^{s s} \times_{P G L(p)} E P G L(p) \rightarrow R^{s s} / P G L(p) \cong \mathcal{M}(n, d)
$$


is a fibration with contractible fibre $\operatorname{EPGL}(p)$ and so induces the isomorphism (13). As morphisms of nonsingular quasi-projective varieties induce maps on cohomology which are strictly compatible with both the Hodge filtration and the weight filtration [5, II3.2.11.1], the isomorphism (13) respects the Hodgestructures. The natural map

$$
R^{s s} \times_{G L(p)} E G L(p) \rightarrow R^{s s} / G L(p) \cong \mathcal{M}(n, d)
$$

is a fibration with fibre $E G L(p) / \mathbf{C}^{*}$ which can be identified with $B \mathbf{C}^{*}$ since $E G L(p)$ is a contractible space on which $\mathbf{C}^{*}$ acts freely. This fibration is cohomologically trivial $\mathbb{1}$, 9.3] and so induces an isomorphism between $H_{G L(p)}^{*}\left(R^{s s}\right)$ and $H^{*}(\mathcal{M}(n, d)) \otimes H^{*}\left(B \mathbf{C}^{*}\right)$, which is an isomorphism of Hodge structures (cf. [5, III 8.1] and [5, III 8.2.10]). Thus by (12) we have

$$
H P_{G L(p)}\left(R^{s s}\right)(x, y)=\frac{1}{1-x y} H P(\mathcal{M}(n, d))(x, y)
$$

Next let us consider, from the point of view of Hodge numbers, the isomorphism (8) between $H_{G L(p)}^{*}(R)$ and $H_{\mathcal{G}}^{*}(\mathcal{C})$ up to some degree tending to infinity with $d$. Since $\mathcal{C}$ is contractible $H_{\mathcal{G}}^{*}(\mathcal{C})$ is canonically isomorphic to $H^{*}(B \mathcal{G})$ where $B \mathcal{G}$ is the classifying space for the gauge group $\mathcal{G}$. By [1] Prop. 2.4] $B \mathcal{G}$ can be identified with a component $\operatorname{Map}_{d}(\Sigma, B U(n))$ of the space of continuous maps $\operatorname{Map}(\Sigma, B U(n))$ from $\Sigma$ to $B U(n)$. By [1, Thm. 2.15] and [1, Prop. 2.20] $H^{*}(B \mathcal{G})$ is freely generated as a polynomial algebra tensored with an exterior algebra by generators

$$
a_{r} \in H^{2 r}(B \mathcal{G}), \quad b_{r}^{j} \in H^{2 r-1}(B \mathcal{G}),
$$

for $1 \leq r \leq n$ and $1 \leq j \leq 2 g$, and by

$$
f_{r} \in H^{2 r-2}(B \mathcal{G})
$$

for $2 \leq r \leq n$, where

$$
a_{r} \otimes 1+\sum_{j=1}^{2 g} b_{r}^{j} \otimes \alpha_{j}+f_{r} \otimes \omega
$$

is the $r$ th Chern class of the universal bundle $\mathcal{V}$ over $B \mathcal{G} \times \Sigma$ and $\{1\},\left\{\alpha_{j}: 1 \leq j \leq 2 g\right\}$ and $\{\omega\}$ are the standard bases for $H^{0}(\Sigma), H^{1}(\Sigma)$ and $H^{2}(\Sigma)$. The universal bundle $\mathcal{V}$ is the pullback to $B \mathcal{G} \times \Sigma$ of the universal bundle over the infinite Grassmannian $B U(n)$ via the evaluation map

$$
B \mathcal{G} \times \Sigma=\operatorname{Map}_{d}(\Sigma, B U(n)) \times \Sigma \rightarrow B U(n) .
$$

We can take the total space $E G L(p)$ for the classifying bundle for $G L(p)$ to be the space of all surjective linear maps from $\mathbf{C}^{\infty}$ to $\mathbf{C}^{p}$ with the obvious action of $G L(p)$ (see $\mathbb{1}$, $\S$ 7] or [24, $\S 3$ ] for further details). If we similarly identify $B U(n)$ with the Grassmannian $G(n, \infty)$ of $n$-dimensional quotients (or equivalently subspaces of codimension $n$ ) of $\mathbf{C}^{\infty}$, then we obtain a map

$$
\theta: R \times_{G L_{p}} E G L(p) \rightarrow \operatorname{Map}_{d}(\Sigma, B U(n))=\operatorname{Map}_{d}(\Sigma, G(n, \infty))
$$

defined as follows. Given an element $f: \Sigma \rightarrow G(n, p)$ of $R$ and an element $e: \mathbf{C}^{\infty} \rightarrow \mathbf{C}^{p}$ of $E G L(p)$ then $\theta(f, e)$ is the map from $\Sigma$ to $G(n, \infty)$ which sends $x \in \Sigma$ to the kernel of the composition of the surjection $e: \mathbf{C}^{\infty} \rightarrow \mathbf{C}^{p}$ with the projection of $\mathbf{C}^{p}$ onto its 
quotient $f(x) \in G(n, p)$. By [15, Lemma10.1] and [15, Cor. 7.4] this map $\theta$ induces an isomorphism

$$
H_{\mathcal{G}}^{*}(\mathcal{C})=H^{*}(B \mathcal{G}) \rightarrow H_{G L(p)}^{*}(R)
$$

up to some degree tending to infinity with $d$, as at (8) above. The pullback via $\theta$ of the universal bundle $\mathcal{V}$ over $B \mathcal{G} \times \Sigma$ corresponds to the pullback via the evaluation map

$$
R \times \Sigma \subseteq \operatorname{Hol}_{d}(\Sigma, G(n, p)) \times \Sigma \rightarrow G(n, p)
$$

of the tautological bundle of rank $n$ over the Grassmannian $G(n, p)$ of $n$-dimensional quotients of $\mathbf{C}^{p}$. It is therefore a holomorphic bundle over $R \times \Sigma$ and its Chern classes are of type $(p, p)$ [11, p.417]. Now the generators $a_{r}, b_{r}^{j}$ and $f_{r}$ of $H^{*}(B \mathcal{G})$ were defined by the equation

$$
c_{r}(\mathcal{V})=\dashv_{\nabla} \otimes \infty+\sum_{\mid=\infty}^{\in\}}\left\lfloor_{\nabla}^{\mid} \otimes \alpha_{\mid}+\{\nabla \otimes \omega\right.
$$

for $c_{r}(\mathcal{V}) \in \mathcal{H}^{*}(\mathcal{B G}) \otimes \mathcal{H}^{*}(\Sigma)$, where $\omega \in H^{2}(\Sigma)$ has Hodge type $(1,1)$ and we may choose the basis $\left\{\alpha_{j}: 1 \leq j \leq 2 g\right\}$ for $H^{1}(\Sigma)$ such that $\alpha_{j}$ has type $(1,0)$ if $1 \leq j \leq g$ and Hodge type $(0,1)$ if $g+1 \leq j \leq 2 g$. Since $\theta^{*}\left(c_{r}(\mathcal{V})\right)=c_{r}\left(\theta^{*} \mathcal{V}\right)$ has Hodge type $(r, r)$ it follows that under the isomorphism (8) the generators $a_{r}$ and $f_{r}$ for $H_{\mathcal{G}}^{*}(\mathcal{C})=H^{*}(\mathcal{C})$ correspond to elements of Hodge type $(r, r)$ and $(r-1, r-1)$ respectively, whereas the generators $b_{r}^{j}$ correspond to elements of Hodge type $(r-1, r)$ if $1 \leq j \leq g$ and $(r, r-1)$ if $g+1 \leq j \leq 2 g$. Thus

$$
H P_{G L(p)}(R)(x, y)=\frac{\prod_{l=1}^{n}\left(1+x^{l} y^{l-1}\right)^{g}\left(1+x^{l-1} y^{l}\right)^{g}}{\left(1-x^{n} y^{n}\right) \prod_{l=1}^{n-1}\left(1-x^{l} y^{l}\right)^{2}}+O\left((x y)^{k(n, d)}\right)
$$

where $k(n, d)$ tends to infinity with $d$. We can now prove

THEOREM 1 The Hodge-Poincaré polynomial of the moduli space $\mathcal{M}(n, d)$, when $n$ and $d$ are coprime, satisfies

$$
H P(\mathcal{M}(n, d))(x, y)=(1-x y) F_{n, d}(x, y)
$$

where $F_{n, d}(x, y)=H P_{G L(p)}\left(R^{s s}\right)(x, y)$ is given by the inductive formula

$$
F_{n, d}(x, y)=\frac{\prod_{l=1}^{n}\left(1+x^{l} y^{l-1}\right)^{g}\left(1+x^{l-1} y^{l}\right)^{g}}{\left(1-x^{n} y^{n}\right) \prod_{l=1}^{n-1}\left(1-x^{l} y^{l}\right)^{2}}-\sum_{\mu \neq \mu_{0}}(x y)^{d_{\mu}} \prod_{1 \leq j \leq P} F_{n_{j}, d_{j}}(x, y) .
$$

Here the sum is over all types $\mu=\left(d_{1} / n_{1}, \ldots, d_{P} / n_{P}\right)$ of bundles of rank $n$ and degree $d$ as described at (11), and $d_{\mu}$ is given by (10).

Remark 2 The inductive formula (18) is to be used even when $n$ and $d$ are not coprime. It is for the identity (17) that $n$ and $d$ need be coprime.

PROOF: We can use equation (18) to define $F_{n, d}(x, y)$ by induction on $n$, because when $n=1$ there are no types $\mu \neq \mu_{0}$. Then by (15) above it suffices to prove that when nand $d$ are coprime

$$
H P_{G L(d+n(1-g))}\left(R(n, d)^{s s}\right)(x, y)=F_{n, d}(x, y)
$$


where we have written $R(n, d)$ for $R$ and $d+n(1-g)$ for $p$ to make explicit the dependence on $n$ and $d$.

First note that

$$
\frac{\prod_{l=1}^{n}\left(1+x^{l} y^{l-1}\right)^{g}\left(1+x^{l-1} y^{l}\right)^{g}}{\left(1-x^{n} y^{n}\right) \prod_{l=1}^{n-1}\left(1-x^{l} y^{l}\right)^{2}}
$$

is independent of $d$ and that

$$
d_{\mu}=\sum_{1 \leq j<i \leq P} n_{i} n_{j}\left(\frac{d_{j}}{n_{j}}-\frac{d_{i}}{n_{i}}+g-1\right)
$$

is unchanged if $\mu=\left(d_{1} / n_{1}, \cdots, d_{P} / n_{P}\right)$ is replaced by $\mu+e=\left(d_{1} / n_{1}+e, \cdots, d_{P} / n_{P}+e\right)$ for any $e \in \mathbf{Z}$. Since $\mu+e$ runs over all types of holomorphic bundles of rank $n$ and degree $d+n e$ as $\mu$ runs over types of holomorphic bundles of rank $n$ and degree $d$ (see $\S 1)$, it follows by induction on $n$ that $F_{n, d}(x, y)$ is unchanged if $d$ is replaced by $d+n e$ for any $e \in \mathbf{Z}$. Now recall from $\oint 1$ that there is a stratification $\left\{S_{\beta}: \beta \in \mathcal{B}\right\}$ of $R$ which is the intersection with $R$ of the stratification of the product of Grassmannians $(G(n, p))^{N}$ described in [14, $\left.\S 16\right]$ and is $G L(p)$-equivariantly perfect outside a subset of dimension $k(n, d)$ where $k(n, d) \rightarrow \infty$ as $d \rightarrow \infty$. By [14, $\S 14]$ the equivariant Morse equalities associated to this stratification can be refined to give the following relation between equivariant Hodge-Poincaré polynomials

$$
H P_{G L(p)}(R)(x, y)=\sum_{\beta \in \mathcal{B}}(x y)^{d_{\beta}} H P_{G L(p)}\left(S_{\beta}\right)(x, y)+O\left((x y)^{k(n, d)}\right)
$$

where $d_{\beta}$ is the complex codimension of $S_{\beta}$ in $R$ (see [14, 14.5]). We noted in $\S 1$ that to every possible type $\mu$ of a holomorphic bundle of rank $n$ and degree $d$ we can associate an index $\beta(\mu)$ such that every stratum of $R$ of codimension less than $k(n, d)$ is indexed by $\beta(\mu)$ for some type $\mu=\left(d_{1} / n_{1}, \ldots, d_{P} / n_{P}\right)$. Moreover $S_{\beta(\mu)}$ then has codimension

$$
d_{\beta_{\mu}}=d_{\mu}
$$

where $d_{\mu}$ is defined by (2), and equivariant cohomology

$$
H_{G L(p)}^{*}\left(S_{\beta(\mu)}\right) \cong \bigotimes_{j=1}^{P} H_{G L\left(p_{j}\right)}^{*}\left(R\left(n_{j}, d_{j}\right)^{s s}\right)
$$

where $p_{j}=d_{j}+n_{j}(1-g)$. This isomorphism of equivariant cohomology is induced by inclusions of subvarieties and subgroups (see the proof of [15, Lemma 12.3]) and therefore respects Hodge structures. Thus

$$
H P_{G L(p)}\left(S_{\beta}\right)(x, y)=\prod_{j=1}^{P} H P_{G L\left(p_{j}\right)}\left(R\left(n_{j}, d_{j}\right)^{s s}\right)(x, y) .
$$

Since $R^{s s}$ is the stratum indexed by $\beta\left(\mu_{0}\right)$ where $\mu_{0}=(d / n, \ldots, d / n)$, combining (16), (19), (20) and (21) yields

$$
\begin{array}{r}
H P_{G L(p)}\left(R^{s s}\right)(x, y)=\frac{\prod_{l=1}^{n}\left(1+x^{l} y^{l-1}\right)^{g}\left(1+x^{l-1} y^{l}\right)^{g}}{\left(1-x^{n} y^{n}\right) \prod_{l=1}^{n-1}\left(1-x^{l} y^{l}\right)^{2}} \\
-\sum_{\mu \neq \mu_{0}}(x y)^{d_{\mu}} \prod_{1 \leq j \leq P} H P_{G L\left(d_{j}+n_{j}(1-g)\right)}\left(R\left(n_{j}, d_{j}\right)^{s s}\right)(x, y)+O\left((x y)^{k(n, d)}\right) .
\end{array}
$$


where $k(n, d) \rightarrow \infty$ as $d \rightarrow \infty$. By induction on $n$ it follows that, given any $k>0$, if $e$ is sufficiently large then

$$
\begin{aligned}
H P_{G L(d+n e+n(1-g))}\left(R(n, d+n e)^{s s}\right)(x, y) & =F_{n, d+n e}(x, y)+O\left((x y)^{k}\right) \\
& =F_{n, d}(x, y)+O\left((x y)^{k}\right)
\end{aligned}
$$

But by (9) and (15), when $n$ and $d$ are coprime, $H P_{G L(d+n e+n(1-g))}\left(R(n, d+n e)^{s s}\right)(x, y)$ is independent of $e$ and hence we must have

$$
H P_{G L(d+n(1-g))}\left(R(n, d)^{s s}\right)(x, y)=F_{n, d}(x, y)
$$

as required.

This theorem enables us to calculate the Hodge numbers of $\mathcal{M}(n, d)$. It also gives us the Hodge numbers of the moduli space $\mathcal{M}_{\Lambda}(n, d)$ of bundles with fixed determinant $\Lambda$ via the following lemma

LEMMA 3 The Hodge-Poincaré polynomials of $\mathcal{M}(n, d)$ and $\mathcal{M}_{\Lambda}(n, d)$ satisfy

$$
H P(\mathcal{M}(n, d))(x, y)=(1+x)^{g}(1+y)^{g} H P\left(\mathcal{M}_{\Lambda}(n, d)\right)(x, y) .
$$

PROOF: Atiyah and Bott show [1, Prop. 9.7] that the determinant map

$$
\mathcal{M}(n, d) \rightarrow \mathcal{M}(1, d)=\mathrm{Jac}_{d}
$$

from $\mathcal{M}(n, d)$ to the Jacobian $\operatorname{Jac}_{d}$ with fibre $\mathcal{M}_{\Lambda}(n, d)$ is a cohomologically trivial fibration and so induces an isomorphism of Hodge structures (cf. [5, III 8.1] and [5, III 8.2.10])

$$
H^{*}(\mathcal{M}(n, d)) \cong H^{*}\left(\mathcal{M}_{\Lambda}(n, d)\right) \otimes H^{*}\left(\mathrm{Jac}_{d}\right) .
$$

The Hodge-Poincaré polynomial of the Jacobian is

$$
H P\left(\mathrm{Jac}_{d}\right)(x, y)=(1+x)^{g}(1+y)^{g},
$$

(for example by Theorem 1 above with $n=1$ ) and so the result follows.

Remark 4 When $d$ is odd then a related formula for the dimensions of the Hodge cohomology groups

$$
H^{p, p}\left(\mathcal{M}_{\Lambda}(2,1)\right) \cap H^{2 p}\left(\mathcal{M}_{\Lambda}(2,1) ; \mathbf{Q}\right)
$$

was found by Balaji, King and Newstead [2]. If, for any nonsingular projective variety $X$, we define

$$
P_{H}(X)(t)=\sum_{p \geq 0} t^{p}\left(\operatorname{dim}_{\mathbf{Q}} H^{p, p}(X) \cap H^{2 p}(X ; \mathbf{Q})\right)
$$

then they show that

$$
P_{H}\left(\mathcal{M}_{\Lambda}(2,1)\right)(t)=\frac{P_{H}(J)\left(t^{3}\right)-t^{g} P_{H}(J)(t)}{(1-t)\left(1-t^{2}\right)}
$$

where $J$ is the Jacobian of $\Sigma$. The proof of Theorem 1 and Lemma 3 above could be modified to give an alternative proof of this result and in principle to give an inductive formula for $P_{H}\left(\mathcal{M}_{\Lambda}(n, d)\right)(t)$ when $n>2$, although the latter would be more complicated 
and would involve looking at the Hodge cohomology of the products of moduli spaces.

In [2] the main object of study was the algebraic cohomology of $\mathcal{M}_{\Lambda}(2,1)$. By proving the analogous formula to (22) for the algebraic cohomology it was shown in 22 that the Hodge conjecture for $\mathcal{M}_{\Lambda}(2,1)$ is valid provided that it holds for the Jacobian $J$, which is true for generic curves $\Sigma$. In [4] the Hodge conjecture for generic $\Sigma$ was proved directly for $\mathcal{M}_{\Lambda}(n, d)$ with $n \geq 2$ and more generally for moduli spaces of parabolic bundles, so for such $\Sigma$ the algebraic cohomology groups of $\mathcal{M}_{\Lambda}(n, d)$ would be given by $P_{H}\left(\left(\mathcal{M}_{\Lambda}(2,1)\right)(t)\right.$.

\section{Calculations}

In this section we use the inductive formula of Theorem 1, together with Lemma 3, to give explicit formulas for the Hodge numbers of the moduli space $\mathcal{M}_{\Lambda}(n, d)$.

COROLLARY 5 The Hodge-Poincaré polynomials of $\mathcal{M}_{\Lambda}(n, d)$ for the following low values of $n$ are:

(a) when $n=2$ and $d$ is odd,

$$
\frac{\left(1+x^{2} y\right)^{g}\left(1+x y^{2}\right)^{g}-x^{g} y^{g}(1+x)^{g}(1+y)^{g}}{(1-x y)\left(1-x^{2} y^{2}\right)}
$$

(b) when $n=3$ and $d \equiv 1,2(\bmod 3)$,

$$
\begin{gathered}
\frac{1}{(1-x y)\left(1-x^{2} y^{2}\right)^{2}\left(1-x^{3} y^{3}\right)}\left(\left(1+x^{2} y^{3}\right)^{g}\left(1+x^{3} y^{2}\right)^{g}\left(1+x y^{2}\right)^{g}\left(1+x^{2} y\right)^{g}\right. \\
-x^{2 g-1} y^{2 g-1}(1+x y)^{2}(1+x)^{g}(1+y)^{g}\left(1+x y^{2}\right)^{g}\left(1+x^{2} y\right)^{g} \\
\left.+x^{3 g-1} y^{3 g-1}\left(1+x y+x^{2} y^{2}\right)(1+x)^{2 g}(1+y)^{2 g}\right) .
\end{gathered}
$$

PROOF: (a) For $n=1$ Theorem 11 shows that for any $d$,

$$
F_{1, d}(x, y)=\frac{(1+x)^{g}(1+y)^{g}}{1-x y}
$$

When $n=2$ and $d=1$ then the unstable types $\mu \neq \mu_{0}$ may each be written as $\mu(r)=$ $(r+1,-r)$ for $r \geq 0$ and from (2) we note that $d_{\mu(r)}=2 r+g$. For $n=2$ and $d=1$ equation (18) states that $F_{2,1}(x, y)$ equals

$$
\frac{(1+x)^{g}(1+y)^{g}\left(1+x^{2} y\right)^{g}\left(1+x y^{2}\right)^{g}}{\left(1-x^{2} y^{2}\right)(1-x y)^{2}}-\sum_{r=0}^{\infty}(x y)^{2 r+g} \frac{(1+x)^{2 g}(1+y)^{2 g}}{(1-x y)^{2}},
$$

which in turn equals

$$
\frac{(1+x)^{g}(1+y)^{g}\left(1+x^{2} y\right)^{g}\left(1+x y^{2}\right)^{g}-(x y)^{g}(1+x)^{2 g}(1+y)^{2 g}}{\left(1-x^{2} y^{2}\right)(1-x y)^{2}} .
$$

Applying Lemma 3 and (17) together with the isomorphism9 yields (a) above.

(b) For the case when $n=2$ and $d=0$, we note that the types $\mu \neq \mu_{0}$ are of the 
form $\mu(r)=(r,-r)$ for $r \geq 1$ with $d_{\mu}=2 r+g-1$. Arguing as in (a) above we see that $F_{2,0}(x, y)$ equals

$$
\frac{(1+x)^{g}(1+y)^{g}\left(1+x^{2} y\right)^{g}\left(1+x y^{2}\right)^{g+1}-(x y)^{g}(1+x)^{2 g}(1+y)^{2 g}}{\left(1-x^{2} y^{2}\right)(1-x y)^{2}} .
$$

Now let $n=3$. Notice that the map $E \mapsto E^{*} \otimes L$, where $L$ is a degree one line bundle over $\Sigma$, induces an isomorphism between the Hodge structures of $\mathcal{M}(3,1)$ and $\mathcal{M}(3,2)$ so that we may set $d=1$ without loss of generality. For $n=3$ the types $\mu \neq \mu_{0}$ fall into three types which we shall refer to as $(1,1,1),(2,1)$ and $(1,2)$.

(i) The $(1,1,1)$-types are

$$
\mu=\left(d_{1}, d_{2}, d_{3}\right) \text { where } d_{1}>d_{2}>d_{3} \text { and } d_{1}+d_{2}+d_{3}=1,
$$

and $d_{\mu}$ equals $2\left(d_{1}-d_{3}\right)+3(g-1)$. Let $r=d_{1}-d_{2}$ and $s=d_{2}-d_{3}$. Then

$$
d_{1}=\frac{1}{3}(2 r+s+1), \quad d_{2}=\frac{1}{3}(-r+s+1)
$$

and so we must have $r-s \equiv 1 \bmod 3$. So the possible values of $r$ and $s$ are given by

$$
(r, s)=(3 k+1,3 l+3) \quad \text { or } \quad(3 k+2,3 l+1) \quad \text { or } \quad(3 k+3,3 l+2)
$$

for $k \geq 0, l \geq 0$. Thus $\sum_{(1,1,1)}(x y)^{d_{\mu}} \prod_{i=1}^{3} F_{1, d_{i}}(x, y)$ equals

$$
\begin{gathered}
\frac{(1+x)^{3 g}(1+y)^{3 g}}{(1-x y)^{3}} \sum_{r-s \equiv 1(3)}^{\infty}(x y)^{2 r+2 s+3 g-3} \\
=\frac{(x y)^{3 g-3}(1+x)^{3 g}(1+y)^{3 g}}{(1-x y)^{3}} \sum_{k=0}^{\infty} \sum_{l=0}^{\infty}\left[(x y)^{2(3 k+3 l+4)}+(x y)^{2(3 k+3 l+3)}+(x y)^{2(3 k+3 l+5)}\right]
\end{gathered}
$$

which, with a little simplifying, becomes

$$
\frac{(x y)^{3 g+3}(1+x)^{3 g}(1+y)^{3 g}}{(1-x y)^{3}\left(1-x^{2} y^{2}\right)\left(1-x^{6} y^{6}\right)} .
$$

(ii) The $(2,1)$-types are $\mu(r)=(r / 2, r / 2,1-r)$ for $r \geq 1$ and $d_{\mu(r)}$ equals $3 r+2 g-4$. Thus the contribution of the (2,1)-types to (18) equals

$$
\frac{(1+x)^{g}(1+y)^{g}}{1-x y} \sum_{r=1}^{\infty}(x y)^{3 r+2 g-4} F_{2, r}(x, y) \text {. }
$$

As $F_{2,0}=F_{2,2 r}$ and $F_{2,1}=F_{2,2 r-1}$ for $r \geq 1$ then this in turn equals

$$
\begin{gathered}
\frac{(1+x)^{g}(1+y)^{g}}{1-x y}\left(F_{2,0}(x, y) \sum_{r=1}^{\infty}(x y)^{6 r+2 g-4}+F_{2,1}(x, y) \sum_{r=1}^{\infty}(x y)^{6 r+2 g-7}\right) \\
=\frac{(1+x)^{g}(1+y)^{g}}{(1-x y)\left(1-x^{6} y^{6}\right)}\left[(x y)^{2 g+2} F_{2,0}(x, y)+(x y)^{2 g-1} F_{2,1}(x, y)\right] .
\end{gathered}
$$

(iii) Similarly the (1,2)-types are given by $\mu(r)=(r,(1-r) / 2,(1-r) / 2)$ for $r \geq 1$ and $d_{\mu(r)}$ equals $3 r+2 g-3$. Arguing as in part (ii) we see that the contribution of the $(1,2)$-types to (18) equals

$$
\frac{(1+x)^{g}(1+y)^{g}}{(1-x y)\left(1-x^{6} y^{6}\right)}\left[(x y)^{2 g} F_{2,0}(x, y)+(x y)^{2 g+3} F_{2,1}(x, y)\right] .
$$

Substituting the terms (23), (24),(25), (26) and (27) into (18) we obtain the final expression (b). 
COROLLARY 6 The $\chi(t)$-characteristic of $\mathcal{M}_{\Lambda}(n, d)$, that is $\operatorname{HP}\left(\mathcal{M}_{\Lambda}(n, d)\right)(t,-1)$, equals

$$
\left(\prod_{r=1}^{n-1}\left(1-(-t)^{r}\right)\left(1-(-t)^{r+1}\right)\right)^{g-1}
$$

The $\chi(t)$-characteristic of $\mathcal{M}(n, d)$ is identically zero.

PROOF: Note from Lemma 3 and (17) that

$$
F_{n, d}(x, y)=\frac{(1+x)^{g}(1+y)^{g} H P\left(\mathcal{M}_{\Lambda}(n, d)\right)(x, y)}{1-x y} .
$$

Substituting this into the recurrence relation (18) we obtain

$$
\begin{gathered}
H P\left(\mathcal{M}_{\Lambda}(n, d)\right)(x, y)=\frac{\prod_{l=2}^{n}\left(1+x^{l} y^{l-1}\right)^{g}\left(1+x^{l-1} y^{l}\right)^{g}}{\prod_{l=2}^{n}\left(1-x^{l} y^{l}\right) \prod_{l=1}^{n-1}\left(1-x^{l} y^{l}\right)} \\
-\sum_{\mu \neq \mu_{0}}(x y)^{d_{\mu}} \frac{(1+x)^{(P-1) g}(1+y)^{(P-1) g}}{(1-x y)^{(P-1) g}} \prod_{1 \leq j \leq P} H P\left(\mathcal{M}_{\Lambda}\left(n_{j}, d_{j}\right)\right)(x, y) .
\end{gathered}
$$

As $P \geq 2$ for each $\mu \neq \mu_{0}$, then setting $x=t$ and $y=-1$ yields the required result for $\mathcal{M}_{\Lambda}(n, d)$. That the $\chi(t)$-characteristic of $\mathcal{M}(n, d)$ is identically zero follows from Lemma 3.

COROLLARY 7 The Euler characteristic and signature of $\mathcal{M}_{\Lambda}(n, d)$ are zero.

PROOF: These are respectively $\chi(-1)$ and $\chi(1)$.

\section{The $\chi(t)$-characteristic}

Let $N=\operatorname{dim}_{\mathbf{C}}\left(\mathcal{M}_{\Lambda}(n, d)\right)=\left(n^{2}-1\right)(g-1)$ and let $c_{r}$ denote the $r$ th Chern class of $\mathcal{M}_{\Lambda}(n, d)$ for $0 \leq r \leq N$. We also introduce here the notation $\bar{g}=g-1$. By the Riemann-Roch Theorem the $\chi(t)$-characteristic may be expressed as an evaluation on the fundamental class $\left[\mathcal{M}_{\Lambda}(n, d)\right]$ of $\mathcal{M}_{\Lambda}(n, d)$. Let $T$ denote the tangent bundle of $\mathcal{M}_{\Lambda}(n, d)$. We then have[22, p. 142] that

$$
\chi(t)=(-1)^{N}\left(\sum_{p=0}^{N} t^{p} \operatorname{ch}\left(\bigwedge^{N-p} T^{*}\right) \operatorname{td} T\right)\left[\mathcal{M}_{\Lambda}(n, d)\right] .
$$

Thus the $\chi(t)$-characteristic which we determined in Corollary6 contains information, though limited, on the Chern numbers of $\mathcal{M}_{\Lambda}(n, d)$. From (28) we know that

$$
\chi(-1)=c_{N}\left[\mathcal{M}_{\Lambda}(n, d)\right]=0 .
$$

More generally, for $2 \leq k \leq N$, we may write $\chi^{(k)}(-1)$ as a linear combination of Chern numbers each of which includes at least one $c_{i}$ with $i>N-2[k / 2]$. (See [22, $\S 3$ ] for details.) For example, [22, 3.7]

$$
\chi^{\prime \prime}(-1)=\frac{1}{24}\left(2 c_{1} c_{N-1}+N(3 N-5) c_{N}\right)\left[\mathcal{M}_{\Lambda}(n, d)\right],
$$


although for large values of $k$ these combinations of Chern numbers rapidly become more complicated. From (30) we see that

$$
c_{N}=0 .
$$

By (31) it follows that $c_{1} c_{N-1}=0$. In fact as $c_{1}=2 n f_{2}$ and since $f_{2}$ generates $H^{2}\left(\mathcal{M}_{\Lambda}(n, d)\right)$ then

$$
c_{N-1}=0
$$

by Poincaré duality. Unfortunately it is not obvious whether we may deduce any further vanishing of the Chern classes from these relations. It was proved by Gieseker [10] that $c_{r}=0$ when $n=2$ and $r>2 g-2$ and it has been conjectured [9] for $n>2$ that $c_{r}=0$ when $r>n(n-1)(g-1)$.

We may rewrite the pairing (29) in terms of pairings involving the generators $a_{r}, b_{r}^{s}$ and $f_{r}$ as follows. If $\gamma_{1}, \cdots, \gamma_{N}$ are the Chern roots of $T$ then (see [13, p.430])

$$
\sum_{p=0}^{N} t^{p} \operatorname{ch}\left(\bigwedge^{N-p} T^{*}\right)=\prod_{p=1}^{N}\left(t+e^{-\gamma_{p}}\right) .
$$

More generally, if

$$
\operatorname{ch} T=\sum_{p=1}^{N} e^{\gamma_{p}}=\sum_{i=1}^{K} \mu_{i} e^{\epsilon_{i}}
$$

where $\mu_{1}, \cdots, \mu_{K}$ and $\epsilon_{1}, \cdots, \epsilon_{K}$ are formal cohomology classes of degrees zero and two respectively, then

$$
\sum_{p=0}^{N} t^{p} \operatorname{ch}\left(\bigwedge^{N-p} T^{*}\right)=(1+t)^{N} \prod_{i=1}^{K}\left(1+\frac{e^{-\epsilon_{i}}-1}{1+t}\right)^{\mu_{i}},
$$

where $(1+y)^{\mu}$ is to be interpreted as the formal power series

$$
\exp (\mu \log (1+y))=1+\mu y+\frac{\mu(\mu-1)}{2 !} y^{2}+\frac{\mu(\mu-1)(\mu-2)}{3 !} y^{3}+\cdots .
$$

As in [8] we now introduce formal cohomology classes $\delta_{k}, W_{k}$ and $\Xi_{k, l}$ as follows. Here $\delta_{1}, \ldots, \delta_{n}$ are formal degree two classes whose sum is zero and whose $r$ th elementary symmetric polynomial equals $a_{r}$ for $r \geq 2$, and $W_{k}$ and $\Xi_{k, l}$ are given by the formulas

$$
W_{k}=\sum_{i=1}^{n} f_{i} \frac{\partial \delta_{k}}{\partial a_{i}}+\sum_{i=2}^{n} \sum_{j=2}^{n} \sum_{s=1}^{g} b_{i}^{s} b_{j}^{s+g} \frac{\partial^{2} \delta_{k}}{\partial a_{i} \partial a_{j}}
$$

and

$$
\Xi_{k, l}=\sum_{s=1}^{g}\left(\sum_{i=2}^{n} b_{i}^{s} \frac{\partial \delta_{k}}{\partial a_{i}}-\sum_{j=2}^{n} b_{j}^{s} \frac{\partial \delta_{l}}{\partial a_{j}}\right)\left(\sum_{i=2}^{n} b_{i}^{s+g} \frac{\partial \delta_{k}}{\partial a_{i}}-\sum_{j=2}^{n} b_{j}^{s+g} \frac{\partial \delta_{l}}{\partial a_{j}}\right)
$$

The tangent bundle $T$ equals $1-\pi_{!}($End $V)$ where $V$ is a universal bundle over $\mathcal{M}_{\Lambda}(n, d)$ and $\pi: \mathcal{M}_{\Lambda}(n, d) \times \Sigma \rightarrow \mathcal{M}_{\Lambda}(n, d)$ is the first projection [1, p.582]. Arguing as in [8, Prop. 10] we find that the Chern character of $T$ equals

$$
\operatorname{ch} T=-\bar{g}+\sum_{k=1}^{n} \sum_{l=1}^{n}\left(\bar{g}+W_{l}-W_{k}-\Xi_{k, l}\right) e^{\delta_{k}-\delta_{l}} .
$$


Let $\lambda_{1}, \ldots, \lambda_{g}$ denote the roots of the equation

$$
x^{g}-x^{g-1}+\frac{1}{2} x^{g-2}-\cdots+\frac{(-1)^{j}}{j !} x^{g-j}+\cdots+\frac{(-1)^{g}}{g !}=0 .
$$

Then [16, p.862]

$$
\sum_{j=1}^{g}\left(\lambda_{j}\right)^{r}= \begin{cases}g & r=0 \\ 1 & r=1 \\ 0 & r \geq 2\end{cases}
$$

and hence

$$
\sum_{j=1}^{g} e^{-\lambda_{j} \Xi_{k, l}}=g-\Xi_{k, l}
$$

since $\left(\Xi_{k, l}\right)^{g+1}=0$. So expression (35) for the Chern character of $T$ may be written as

$$
\operatorname{ch} T=-\bar{g}+\sum_{k=1}^{n} \sum_{l=1}^{n}\left(W_{l}-W_{k}-1\right) e^{\delta_{k}-\delta_{l}}+\sum_{k=1}^{n} \sum_{l=1}^{n} \sum_{j=1}^{g} e^{\delta_{k}-\delta_{l}-\lambda_{j} \Xi_{k, l}},
$$

and using (32) we find that $\sum_{p=0}^{N} t^{p} \operatorname{ch}\left(\Lambda^{N-p} T^{*}\right)$ equals

$$
(1+t)^{N-n(n-1) g} \prod_{k \neq l}\left(1+\frac{e^{\delta_{k}-\delta_{l}}-1}{1+t}\right)^{W_{k}-W_{l}-1} \prod_{j=1}^{g}\left(t+e^{\delta_{l}-\delta_{k}+\lambda_{j} \Xi_{k, l}}\right)
$$

which with some simplifying becomes

$$
(1+t)^{N} \prod_{k \neq l}\left(1+\frac{e^{\delta_{k}-\delta_{l}}-1}{1+t}\right)^{W_{k}-W_{l}+\bar{g}} \exp \left\{\frac{\Xi_{k, l}}{1+e^{\delta_{k}-\delta_{l}} t}\right\} .
$$

Now the non-zero Pontryagin roots of $\mathcal{M}_{\Lambda}(n, d)$ are $\left(\delta_{k}-\delta_{l}\right)^{2}$ for $k<l$ and each has multiplicity $2 g-2$ [8, Lemma 17]. We also know that

$$
c_{1}=2 n f_{2}=2 \sum_{k<l}\left(W_{l}-W_{k}\right)\left(\delta_{k}-\delta_{l}\right)-\Xi_{k, l} .
$$

Hence

$$
\operatorname{td} T=\prod_{k<l} e^{\left[\left(W_{l}-W_{k}\right)\left(\delta_{k}-\delta_{l}\right)-\Xi_{k, l}\right]}\left(\frac{\delta_{k}-\delta_{l}}{2 \sinh \frac{1}{2}\left(\delta_{k}-\delta_{l}\right)}\right)^{2 g-2} .
$$

We have thus shown, with some arranging, that

PROPOSITION 8 The pairing

$$
\begin{array}{r}
\left\{\prod_{k<l}\left(t+e^{X_{k, l}}\right)^{\bar{g}}\left(t+e^{X_{l, k}}\right)^{\bar{g}}\left(\frac{X_{k, l}}{2 \sinh \frac{1}{2}\left(X_{k, l}\right)}\right)^{2 \bar{g}}\left(1+\frac{(1-t)\left(e^{X_{k, l}}-1\right)}{1+t e^{X_{k, l}}}\right)^{W_{k}-W_{l}} \times\right. \\
\left.\times \exp \left(\frac{\Xi_{k, l}\left(1-t^{2}\right)}{\left(1+t e^{X_{k, l}}\right)\left(1+t e^{X_{l, k}}\right)}\right)\right\}\left[\mathcal{M}_{\Lambda}(n, d)\right],
\end{array}
$$

where $X_{k, l}=\delta_{k}-\delta_{l}$, is equal to

$$
\frac{1}{(1+t)^{(n-1)(g-1)}}\left(\prod_{r=1}^{n-1}\left(1-(-t)^{r}\right)\left(1-(-t)^{r+1}\right)\right)^{g-1} .
$$


Assume now that $n=2$ and let $\alpha=2 f_{2}, \beta=-4 a_{2}$ and $\gamma=2 \sum_{s=1}^{g} b_{2}^{s} b_{2}^{s+g}$ denote Newstead's generators [20] for the subring of $H^{*}\left(\mathcal{M}_{\Lambda}(2,1)\right)$ invariant under the induced action of the mapping class group of $\Sigma$. In terms of these generators the previous proposition reads as (with some simplification),

COROLLARY 9 When $n=2$ the pairing of

$\left(t+e^{X}\right)^{\bar{g}}\left(t+e^{-X}\right)^{\bar{g}}\left(\frac{X}{e^{X / 2}-e^{-X / 2}}\right)^{2 \bar{g}}\left(1+\frac{(1-t)\left(e^{X}-1\right)}{1+t e^{X}}\right)^{D} \exp \left\{\frac{\xi\left(1-t^{2}\right)}{\left(1+t e^{X}\right)\left(1+t e^{-X}\right)}\right\}$

with $\left[\mathcal{M}_{\Lambda}(n, d)\right]$ equals $\left(1-t^{2}\right)^{g-1}$ where

$$
X=X_{1,2}=\delta_{1}-\delta_{2}=\sqrt{\beta}, \quad D=W_{1}-W_{2}=-(\alpha \beta+2 \gamma) \beta^{-3 / 2}, \quad \xi=\Xi_{1,2}=2 \gamma / \beta .
$$

Remark 10 In the rank two case Thaddeus [23] showed that the intersection pairings $\eta\left[\mathcal{M}_{\Lambda}(2,1)\right]$, where $\eta \in H^{3 g-3}\left(\mathcal{M}_{\Lambda}(2,1)\right)$, can all be expressed in terms of pairings just involving $\alpha, \beta$ and $\gamma$. In turn these pairings may be expressed in terms of intersection pairings of moduli spaces over Riemann surfaces of lower genus involving just $\alpha$ and $\beta$ by the equation

$$
\left(\alpha^{m} \beta^{n} \gamma^{p}\right)\left[\mathcal{M}_{\Lambda}^{g}(2,1)\right]=2 g\left(\alpha^{m} \beta^{n} \gamma^{p-1}\right)\left[\mathcal{M}_{\Lambda}^{g-1}(2,1)\right]
$$

He then determined the pairings involving just $\alpha$ and $\beta$ from the Verlinde formula.

Information concerning pairings involving $\alpha, \beta$ and $\gamma$ is contained in Corollary 9 but is less tractable. Not all the pairings involving $\alpha, \beta$ and $\gamma$ may be determined from the $\chi(t)$-characteristic but low genus calculations suggest that sufficient information is present to prove Newstead's and Ramanan's conjecture (that $\beta^{g}=0$ ) [20, p.344]. Such a proof would be of some interest historically since the $\chi(t)$-characteristic of $\mathcal{M}_{\Lambda}(2,1)$ was known as early as 1975 [18], soon after the Newstead-Ramanan conjecture was made, and long before the Verlinde formula was proved from which Thaddeus derived his proof.

\section{References}

[1] M.F.Atiyah and R.Bott The Yang-Mills equations over Riemann surfaces Philos. Trans. Roy. Soc. London Ser. A 308 (1982) 523-615.

[2] V.Balaji, A.D.King and P.E.Newstead Algebraic cohomology of the moduli space of rank 2 vector bundles on a curve Topology 36 (1997) 567-577.

[3] S. del Baño Rollin The motive of some moduli spaces of vector bundles over a curve alg-geom/9501013

[4] I. Biswas \& M.S. Narasimhan Hodge classes of moduli spaces of parabolic bundles over the general curve J. Alg. Geom. 6 (1997) 697-715.

[5] P.Deligne, Théorie de Hodge I Actes du Congrés International des Mathématiciens Nice 1970, II Publ. Math. IHES 40 1971, III Publ. Math. IHES 441974. 
[6] P.Deligne Poids dans la cohomologie des variétés algébriques Actes du Congrés International des Mathématiciens Vancouver 1974.

[7] U.V.Desale and S. Ramanan Poincaré polynomials of the variety of stable bundles Math. Ann. 216 (1975) 233-244.

[8] R.A.Earl The Mumford relations and the moduli of rank three stable bundles Compositio Math. 109 (1997) 13-48.

[9] R.A.Earl and F.C.Kirwan The Pontryagin rings of moduli spaces of arbitrary rank holomorphic bundles over a Riemann surface alg-geom/9709012

[10] D.Gieseker A Degeneration of the Moduli Spaces of Stable Bundles J. Differential Geom. 19 (1984) 173-206.

[11] P.Griffiths and J.Harris Principles of Algebraic Geometry Interscience Series of Texts, Monographs and Tracts (Wiley) 1978.

[12] G.Harder and M.S.Narasimhan On the cohomology groups of moduli spaces of vector bundles over curves Math. Ann. 212 (1975) 215-248.

[13] R.Hartshorne Algebraic geometry GTM 52 Springer-Verlag New York-HeidelbergBerlin 1977.

[14] F.C.Kirwan Cohomology of quotients in symplectic and algebraic geometry Math. Notes vol. 31 Princeton Univ. Press, Princeton, NJ 1985.

[15] F.C.Kirwan On spaces of maps from Riemann surfaces to Grassmannians and applications to the cohomology of moduli of vector bundles Ark. Math. 24 (1986) 221-275.

[16] F.C.Kirwan Cohomology rings of moduli spaces of bundles over Riemann surfaces J. Amer. Math. Soc. 5 (1992) 853-906.

[17] D.B.Mumford, J.Fogarty and F.C.Kirwan Geometric Invariant Theory Ser. Modern Surveys Math. 34 Third Edition 1994.

[18] M.S.Narasimhan and S.Ramanan Generalised Prym varieties as fixed points J. Indian Math. Soc. 39 (1975) 1-19.

[19] P.E.Newstead Introduction to moduli problems and orbit spaces Tata Inst. Lect. 51 1978.

[20] P.E.Newstead Characteristic classes of stable bundles of rank 2 over an algebraic curve Trans. Amer. Math. Soc. 169 (1972) 337-345.

[21] P.E.Newstead Unpublished work and private communication.

[22] S.M.Salamon On the cohomology of Kähler and hyper-Kähler manifolds Topology 35 137-155.

[23] M.Thaddeus Conformal field theory and the cohomology of the moduli space of stable bundles J. Differential Geom. 35 (1992) 131-149. 
[24] J-L.Verdier, J.Le Potier et al. Module des Fibrés Stables sur les Courbes Algebriqués Progress in Mathematics Vol.54 (Birkhäuser) 1985.

[25] E.Verlinde Fusion rules and modular transformations in 2d conformal field theory, Nuclear Phys. B 300 (1988) 360-376.

Mathematical Institute, 24-29 St. Giles, Oxford OX1 3LB, England

Email: earl@maths.ox.ac.uk, kirwan@maths.oxford.ac.uk 